\title{
Inovação responsável através de fronteiras: tensões, paradoxos e possibilidades
}

\author{
Responsible innovation across borders: tensions, paradoxes and possibilities
}

\section{Phil Macnaghten}

Professor de Tecnologia e Desenvolvimento Internacional, Universidade de Wageningen, Holanda

philip.macnaghten@wur.nl

\section{Richard Owen}

Professor de Gestão da Inovação

Estratégica, Universidade de Exeter, Escola de Negócios

R.J.Owen@exeter.ac.uk

\section{Jack Stilgoe}

Lecturer, University College London,

Departamento de Estudos da Ciência e da

Tecnologia

j.stilgoe@ucl.ac.uk

\section{Brian Wynne}

Professor de Estudos da Ciência, Universidade de Lancaster, Centro de Pesquisas sobre Mudanças Ambientais (CSEC)

b.wynne@lancaster.ac.uk

\section{Adalberto Azevedo \\ Professor Adjunto, Universidade Federal do ABC, Centro de Engenharia e Ciências Sociais Aplicadas adalberto.azevedo@ufabc.edu.br}

\section{André Luiz Sica de Campos}

Professor Doutor, Universidade Estadual de Campinas, Faculdade de Ciências Aplicadas andre.campos@fca.unicamp.br

\section{Jason Chilvers}

Senior Lecturer em Ambiente, Universidade de East Anglia, Ciência e Sociedade na Escola de Ciências Ambientais Jason.Chilvers@uea.ac.uk

\section{Renato Dagnino}

Professor Titular, Universidade Estadual de Campinas, Departamento de Política Científica e Tecnológica rdagnino@ige.unicamp.br

\section{Gabriela Di Giulio}

Professora Doutora, Universidade de São Paulo, Faculdade de Saúde Pública, Departamento de Saúde Ambiental ggiulio@usp.br

\section{Emma Frow}

Assistant Professor, Arizona State University, School of Biological \& Health Systems Engineering e School for the Future of Innovation in Society emma.frow@asu.edu

\section{Brian Garvey}

Research Associate, Universidade de Strathclyde, Escola de Negócios

brian.garvey@strath.ac.uk

\section{Christopher Groves}

Research Associate, Universidade de Cardiff, Escola de Clências Sociais

GrovesC1@cardiff.ac.uk

\section{Sarah Hartley}

Research Fellow, Universidade de Nottingham, Escola de Biociências Sarah.Hartley@nottingham.ac.uk

\section{Marcelo Knobel}

Professor Titular, Universidade Estadual de Campinas, Instituto de Física

knobel@ifi.unicamp.br

\section{Elizabete Kobayashi}

Pesquisadora Pos-doutoranda, Universidade Federal de São Carlos

elizabetekobayashi@gmail.com

\section{Markku Lehtonen}

Chargé de recherche no Centro de Pesquisa em Sociologia Pragmática e Reflexiva (GSPR), Escola de Estudos Avançados em Ciências Sociais (EHESS), Paris.

ESSEC Business School, Cergy-Pontoise markku.lehtonen@ehess.fr

\section{Javier Lezaun James Martin}

Lecturer em Governança de Ciência e Tecnologia, School of Anthropology and Museum Ethnography, Universidade de Oxford

javier.lezaun@insis.ox.ac.uk

\section{Leonardo Freire de Mello}

Professor Adjunto do Bacharelado em Planejamento Territorial, Universidade Federal do ABC, Centro de Engenharia, Modelagem e Ciências Sociais Aplicadas, Programa de Pós-Graduação em Planejamento e Gestão do Território leonardo.mello@ufabc.edu.br

\section{Marko Monteiro}

Professor Doutor, Universidade Estadual de Campinas, Departamento de Política Científica e Tecnológica markosy@uol.com.br

\section{Janaina Pamplona da Costa}

Professora Doutora, Universidade Estadual de Campinas, Departamento de Política

Científica e Tecnológica

jpamplona@ige.unicamp.br

\section{Camila Rigolin}

Professora Adjunta, Universidade Federal de São Carlos, Centro de Educação e Ciências Sociais

camilacarneiro.dias@gmail.com

\section{Bruno Rondani}

Coordenador, Fundação Getúlio Vargas, Grupo de Estudos em Private Equity and Venture Capital bruno.rondani@wenovate.org

\section{Margarita Staykova}

Lecturer, Universidade de Durham, Departamentos de Física e Química margarita.staykova@durham.ac.uk

\section{Renzo Taddei}

Professor Adjunto, Universidade Federal de São Paulo, Instituto do Mar renzo.taddei@unifesp.br

\section{Chris Till}

Senior Lecturer em Ciências Sociais, Leeds Beckett University

c.till@leedsbeckett.ac.uk 


\section{David Tyfield}

Reader, Universidade de Lancaster, Centro

Lancaster de Meio Ambiente

d.tyfield@lancaster.ac.uk

\section{Léa Velho}

Professora Titular Universidade Estadual de Campinas, Departamento de Política Científica e Tecnológica

velho@ige.unicamp.br

\section{Sara Wilford}

Senior Lecturer e Research Fellow, Universidade de De Montfort

sara@dmu.ac.uk
Resumo: Em março de 2014, um grupo de jovens pesquisadores do estado de São Paulo e do Reino Unido encontraram-se na Universidade de Campinas para participar do Workshop "Inovação Responsável e a Governança de Tecnologias Socialmente Controversas". Este texto apresenta as reflexões e observações mais importantes das discussões travadas ali, com atenção especial para o discurso da inovação responsável, pensada a partir de uma perspectiva comparativa. O texto descreve ainda algumas das tensões, paradoxos e oportunidades importantes que emergiram ao longo dos 3 dias de workshop.

Palavras-chave: Inovação responsável; Economia política; Invisibilidades; Afeto e cuidado.

Abstract: In March 2014 a group of early career researchers and academics from São Paulo state and from the UK met at the University of Campinas to participate in a workshop on 'ResponsibleInnovation and the Governance of Socially Controversial Technologies'. In this Perspective we describe key reflections and observations from the workshop discussions, paying particular attention to the discourse of responsible innovation from a cross-cultural perspective. We describe a number of important tensions, paradoxes and opportunities that emerged over the three days of the workshop.

Keywords: Responsible innovation; Political economy; Invisibilities; Affect and care.
Inovação (e Pesquisa) Responsável (IR) ${ }^{1}$ é um poderoso discurso emergente na política científica, particularmente na Europa. Apesar de ser interpretativamente flexível, enquadramentos de IR vêm se desenvolvendo até o presente principalmente na Europa e na América do Norte, construindo um quadro conceitual promissor para tornar processos de IR responsivos aos desafios sociais em contextos de inevitáveis incertezas, ambiguidades e de novas perguntas criadas pela inovação (OWEN; MACNAGHTEN; STILGOE, 2012; von SCHOMBERG, 2013).

Apesar da globalização, esses enquadramentos vêm até o momento orientando relações entre ciência-inovação-sociedade do Norte global, buscando deslocar a avaliação de ciência e tecnologia para além dos riscos antecipados e os benefícios de mercado. O quadro conceitual de "antecipação, inclusão, reflexividade e responsividade" desenvolvido pelos autores sugere, por exemplo, que a IR pode ser implementada através do desenvolvimento de capacidades institucionais que ajudem pesquisadores a antecipar os possíveis impactos e implicações futuras, que abram tais perguntas para um diálogo mais amplo e inclusive, que encorajem uma reflexão sobre as motivações para e as possíveis

\footnotetext{
${ }^{1}$ No original: Responsible Research and Innovation - RRI
}

implicações da pesquisa, usando tais processos para influenciar o processo de IR ele mesmo de forma responsiva (OWEN; BESSANT; HEINTZ, 2013; STILGOE; OWEN; MACNAGHTEN, 2013).

Mas como a IR poderia ser enquadrada em outras partes do mundo, especialmente no Sul global? Além disso, tais enquadramentos nortistas da IR viajam e são traduzíveis além de fronteiras, e devemos fazer isso? Em março de 2014, 10 jovens pesquisadores (das ciências naturais e sociais) do estado de São Paulo e 11 do Reino Unido se reuniram na Universidade Estadual de Campinas para participar de um workshop sobre a "Inovação Responsável e a Governança de Tecnologias Socialmente Controversas". O workshop, que contou ainda com 3 acadêmicos sêniores de ambos países, buscou fomentar a interação, o aprendizado e a colaboração. Esse trabalho sintetiza algumas reflexões e observações dos participantes, que debateram a IR desde uma perspectiva intercultural.

Se a IR vai fazer uma diferença positiva num mundo que se globaliza de forma acelerada, ela precisa garantir que haja lugar para o Sul global no coração do desenvolvimento do seu discurso, e não como uma ideia secundária, ou um estudo de caso comparativo. Relações Norte-Sul podem tornar-se rapidamente hierarquizadas, incluindo aquelas de produção de 
conhecimento, onde o Sul tende a ser representado como consumidor passivo de conhecimento produzido no Norte. Através do engajamento com o Sul global e suas necessidades específicas e diferenciadas, torna-se explícito que a IR vai ter que ser 'responsável' de formas que não são imediatamente prioritárias para as nações mais desenvolvidas do norte (especialmente os EUA e a UE), onde o discurso da IR tem se desenvolvido com mais força. A IR vai precisar se localizar em um conjunto distinto de debates a respeito do desenvolvimento urbano e econômico, de reformas institucionais, da formação de capacidades, transições e responsabilidade social. As formas pelas quais a IR intersecta com, ou é desafiada por conceitos paralelos como "inclusão social” (DAGNINO, 2012), ou iniciativas como as de Buen Vivir que buscam construir um desenvolvimento em linha com o passado indígena de um país (GUDYNAS, 2011), ou narrativas associadas com a abertura de mercados na chamada "base da pirâmide" (PRAHALAD, 2006), ou aqueles que argumentam por inovações mais "inclusivas", "grassroots" ou "empáticas" - permanecem questões em aberto.

Ao considerar a IR a partir de uma perspectiva do sul, talvez o maior risco seja o que os participantes brasileiros chamaram de "coerção ideológica": isto é, impingir um conceito que tem tido um ar "nortista" (e em termos de políticas públicas, europeu) ao sul global com pouca consideração pelo contexto e pelas premissas embutidas na IR, sendo esta um artefato político do Norte (por exemplo: em termos culturais, políticos, econômicos, demográficos, de governança e estruturas de poder, de arranjos institucionais e de relações entre ciência e sociedade). A IR é interpretativamente flexível, culturalmente enquadrada e politicamente localizada. Se a IR no seu enquadramento nortista busca, de forma ampla, construir processos e instituições públicas e políticas que prestem atenção e sejam responsivas de forma sistemática para os aspectos políticos e sociais da IR, tais dimensões políticas e sociais não podem ser ignoradas no Sul global. A IR, na sua constituição nortista, possui uma base normativa que advoga por uma "ordem sociotécnica diferente para o porvir", uma que "acene para uma relação entre ciência e sociedade que seja mais inclusiva, democrática e equitativa" (van OUDHEUSDEN, 2014, p. 72). Mas, de uma perspectiva do Sul (apesar da grande heterogeneidade que caracteriza os países, regiões, municipalidades e instituições do Sul global), as premissas do Norte a respeito do que seja (ou deveria ser) essa ordem sociotécnica e essas relações entre ciência e sociedade são, em termos mais otimistas, ingênuas. Em termos pessimistas, elas podem transformar a IR em um novo instrumento daquilo que um participante brasileiro chamou de "neo-colonização" intelectual - já que poderia inadvertidamente reproduzir ou reforçar relações de dependência que estão longe de ser emancipatórias para o Sul global. Dessa forma, consideramos que a IR é um lócus de conflitos constitutivos e discursivos que, ao serem explorados, descrevem algumas tensões, paradoxos e oportunidades que emergiram no decorrer dos 3 dias do workshop.

\section{As múltiplas produções e circulações da inovação responsável}

O workshop sensibilizou os participantes para as formas através das quais definições, performances e possibilidades da IR variam através de culturas políticas e estados-nação. Precisamos ser sensíveis para as particularidades sócio-políticas dos contextos nos quais a IR emergiu como um discurso de política científica na Europa e na América do Norte e como, sob quais condições e com quais efeitos esses discursos viajam para contextos não-ocidentais.

Diversos participantes perceberam os enquadramentos atuais da IR como ligados a um conjunto de prioridades, valores e preocupações europeias. Esses incluem um foco em tecnologias emergentes e potencialmente disruptivas como biotecnologia, nanotecnologia, biologia sintética, robótica e geoengenharia; a ambição de influenciar as trajetórias da ciência e da inovação com base em valores europeus; seu uso como um veículo de mudança da "governança do risco" para a "governança da inovação"; e suas ambições de reconfiguração e aumento das responsabilidades e capacidades de cientistas e inovadores para que eles possam cuidar melhor do futuro através de treinamentos sistemáticos nas universidades e pelo encorajamento da troca e colaboração entre os setores acadêmico e industrial (FELT et al., 2007; OWEN; BESSANT; HEINTZ, 2013; STILGOE; OWEN; MACNAGHTEN, 2013).

De um ponto de vista brasileiro (e do Sul global), essas premissas parecem menos robustas. Privilegiar tecnologias emergentes pode ser menos relevante $\mathrm{e}$, de fato, tais enquadramentos podem encobrir precisamente as questões problemáticas associadas com a IR que uma política de IR efetiva deve iluminar e enfrentar. Um foco nas tecnologias emergentes pode sugerir, por exemplo, que um caso prioritário para o Brasil seja os biocombustíveis de 2a geração. Ainda que importante, isso possivelmente teria pouco impacto na vida cotidiana da maior parte dos brasileiros, para os quais há desafios maiores ligados ao desenvolvimento socioeconômico e urbano do que a inovação tecnológica de ponta (a não ser que a IR consiga aproximar os dois). Tal abordagem esconderia a separação entre uma ênfase na ciência e inovação 
(e política) no Norte e a relevância dessa ciência e inovação (e política) para a maioria da população no Sul global. Portanto, devemos considerar melhor quais questões a IR deve enfrentar e que essa perspectiva é ao mesmo tempo contextualizada por necessidades locais e preocupada com o impacto global e desigual de práticas de inovação (GUPTA, 2012).

Com isso em mente, devemos então localizar e engajar a IR em contextos, culturas e práticas locais. Isso demanda que prestemos atenção a, por exemplo, formas de conhecimento locais, tradicionais e não-ocidentais; contextos sociais e religiosos (incluindo questões de gênero, como sistemas patrilineares de comportamento e poder); e direitos de propriedade e padrões de posse em geral. Por exemplo, como os conceitos feministas de cuidado que enquadram as concepções do Norte de IR seriam traduzíveis para esses contextos tão distintos? Foi também reconhecido que, ainda que a IR tenha se desenvolvido no contexto de, e como resposta de política pública a, formas controversas ou questionáveis de inovação tecnológica, há que se reconhecer a variedade enorme de inovações sociais que ocorrem no Sul global, direcionadas especificamente para objetivos sociais e/ou ambientais, por vezes como ajuste-resposta a inovações tecnológicas desenhadas ou escolhidas de forma inadequada. A IR precisa considerar seu papel, se é que ele existe, na acomodação e encorajamento dessas inovações, gerando recomendações para uma política de inovação que seja mais focada em problemas sociais, e menos em alta tecnologia.

Os participantes discutiram a ideia de que as formulações atuais do Norte tendem a representar a IR como algo que é feito à ciência, tecnologia e inovação: tanto como experimento quanto como uma inovação em processos e relações de governança. Por sua vez, isso demanda que prestemos atenção para os contextos e significados da governança em diferentes partes do mundo, incluindo questões (locais, nacionais e regionais) de efetividade e eficiência, de democracia representativa, accountability/responsabilização final, sustentabilidade ambiental, equidade e justiça, respeito pela lei, os limites do capitalismo, a necessidade de consumir menos, assim como o desejo e aceitação públicos. Instituições e suas capacidades organizacionais, suas culturas políticas e regulatórias, suas culturas de clima social e de risco são sítios importantes a partir dos quais podemos observar a abertura ou fechamento para a governança da inovação, e não podemos presumir que sejam iguais para todo o globo. A IR precisa assim incluir questões de economia política e de relações de poder entre redes e atores de contextos geográficos diferentes, cujos públicos e instituições façam parte dos sistemas de inovação que contribuem para novas formas de ordenamentos sociais, políticos e técnicos.

\section{(In)visibilidades e emergência}

O workshop colocou em debate qual seria o objeto da $I R$ : sobre o que exatamente se está sendo responsável? A questão central aqui é garantir que a IR não restrinja a definição dos problemas nem torne incomensuráveis ou invisíveis enquadramentos distintos dos problemas. Ontologias alternativas, formas distintas de definição de problemas, prioridades e questões, e possíveis trajetórias alternativas de inovação e conhecimento nascem condenadas por falta de abordagens reflexivas que desafiem formas estabelecidas de definição e naturalização dos objetos de análise. Tomando o exemplo dos insetos geneticamente modificados (GM; uma tecnologia emergente no Reino Unido e no Brasil), como se podem criar as condições para garantir que a IR dê espaço para a discussão de políticas públicas alternativas ao uso de tais insetos na agricultura? Insetos geneticamente modificados podem ou não ter papel importante na abordagem de desafios como a segurança alimentar e a proteção ambiental, mas precisam ser pensadas em conjunção com alternativas que transcendam considerações econômicas de curto prazo. Com a mudança do foco, da "inovação responsável" para a "governança responsável”, os participantes se sensibilizaram ao risco da aceitação tácita de que a inovação é o único caminho, ou o preferencial, para a resolução de desafios sociais e políticas públicas problemáticas. Para que qualquer formulação de IR seja transparente e capaz de mostrar-se eficaz, é essencial que abordagens alternativas mantenham-se visíveis, bem como suas conexões com economias políticas e contextos sociotécnicos mais amplos - especialmente em razão do fato de que fatores políticos e econômicos podem obstruir, desviar ou aniquilar opções alternativas e trajetórias de inovação diferentes.

Em alguns casos, talvez seja preferível não gerar inovação, ou pelo menos inovar de formas que desafiem as formas ocidentais dominantes de compreensão do desenvolvimento e da modernização. Alguns participantes apontaram que, mesmo em termos estritamente econômicos, a inovação não é necessariamente a melhor forma de estímulo à economia. Outros apontaram que inovação não deve ser tratada como uma 'coisa', e que modelos de inovação que incluam abordagens do tipo imitação, adaptação ou bricolagem, ou a partir das bases sociais e populares, ou indígenas (e ainda alternativas ao crescimento como formas de desenvolvimento), como forma de gerar valor e suprir necessidades sociais, permanecem influentes no Brasil e outros países em desenvolvimento. A forma como processos de inovação são entendidos e emergem nos países do Sul coloca um desafio real à IR, em termos de manter-se eficaz e, ao mesmo tempo, manter visível a sua característica de "processo em curso." 
Ainda que a reflexão (e a reflexividade) tenham sido elementos importantes do discurso de IR, uma necessidade específica identificada diz respeito a suas intersecções com o mundo do emprego, trabalho e capital, incluindo a ponderação de até que ponto as consequências potenciais da inovação para os mercados de trabalho (incluindo as condições de trabalho) podem ser incluídas nas formas como a IR é pensada (como na bioeconomia do Brasil). O que, por exemplo, constitui o trabalho de fazer-se responsável? Este elemento foca nossa atenção a questões ligadas ao trabalho emocional/afetivo, a distribuição de responsabilidades e as restrições e pressões institucionais associadas ao "ser responsável". As discussões sobre o trabalho também devem considerar os impactos das transformações das práticas laborais na força de trabalho. Em particular, como poderiam as pessoas que implementam abordagens de IR nas indústrias envolverem (como atores diretos) a grande quantidade de brasileiros que não participam da economia formal e que, como consequência, permanecem economicamente invisíveis?

\section{Questões de economia política}

Os participantes observaram que é necessário ir além da consideração da responsabilidade no nível dos atores ou localidades individuais, e de se considerar as responsabilidades de forma mais sistêmica, de modo a considerar a IR dentro de suas economias políticas mais amplas. A análise de algumas destas dimensões mais amplas e sistêmicas - incluindo o poder corporativo, o apoio político e institucional acrítico para a ciência e a tecnologia, políticas neoliberais, estruturas e modos capitalistas de produção e extração do capital - revela facilmente a existência de irresponsabilidades sistêmicas associadas a poderosas forças geradoras de inovação. Independentemente da existência ou inexistência de formas de "controle" de tais qualidades sistêmicas da "irresponsabilidade", estas devem ser estudadas e entendidas, como passo necessário na direção de uma possível "transformação em direção à responsabilidade".

Os biocombustíveis no Brasil, por exemplo, são frequentemente apresentados como um caso de inovação responsável e sustentável. O Brasil é apresentado como não apenas tendo produzido o programa de biocombustíveis mais desenvolvido e integrado do planeta, como também tendo criado um sistema de produção de bioetanol de cana-de-açúcar que tem baixa emissão de carbono, mitiga de forma eficiente as emissões de gases de efeito estufa (se comparado com biocombustíveis produzidos a partir de outras culturas), não tem impactos acentuados sobre os ecossistemas e representa uma das formas mais promissoras de alcance do desenvolvimento sustentável (ver GOLDEMBERG,
2007; SORDA; BANSE; KEMFERT, 2010; WALTER et al., 2011). No entanto, uma avaliação mais detalhada revela a existência de vários problemas sistêmicos, que vão dos problemas ligados às condições de trabalho dos cortadores de cana-de-açúcar às formas como o apoio governamental aos biocombustíveis na área de transportes condicionou os processos produtivos a trajetórias tecnológicas e econômicas particulares, o que naturalmente descarta formas de inovação potencialmente distintas, mais sustentáveis e mais socialmente justas - neste caso dificultando o estímulo a formas de mobilidade urbana alternativas e não baseadas no uso de automóveis, e desta forma falhando em atuar sobre os níveis dramáticos de congestionamento de tráfego, poluição ambiental, falta de infraestrutura e transporte público de má qualidade, fatos comuns nas maiores cidades brasileiras (DA MATTA, 2010). Apenas em São Paulo - como ilustração da obsessão brasileira implacável por automóveis - estima-se que mais de mil novos automóveis sejam adicionados à malha viária diariamente (MICHENER, 2014).

Estes fatos suscitam questionamentos importantes sobre as escalas nas quais pensamos a responsabilidade no contexto da inovação, e a economia política nas quais estas estão localizadas. E desafiam-nos a compreender - e intervir - nas formas técnicas, sociais, institucionais e culturais de inovação que são continuamente tecidas e enredadas de formas aparentemente indistintas. O paradoxo é que não há um ator em controle, mas todos estão implicados, têm algum grau de agência, e desta forma são responsáveis e estão interconectados através de redes complexas, em múltiplas escalas, e de formas variáveis. A IR não pode ser materializada através de atores, organizações ou instituições isoladas, uma vez que os produtos de tais redes são gerados através de combinações interativas que facilmente transcendem os atores individuais. No entanto, o fato de todos estarmos implicados não significa a existência de horizontalidade, mas que as hierarquias estão interconectadas em redes complexas, e em múltiplas escalas. De fato, alguns participantes questionaram até que ponto se faz necessária a vinculação entre as ideias de responsabilidade e a governança democrática com mecanismos participativos de gestão da inovação. Outras formas de construção e agenciamento da responsabilidade precisam fazer parte do universo empírico em questão.

\section{Afeto, cuidado e capacidades}

Questões ligadas aos afetos foram também debatidas no workshop, demonstrando a importância da contribuição das emoções na formulação da IR. Tal consideração da dimensão afetiva problematizou 
formulações estabelecidas que enquadram os aspectos normativos da IR (como o "cuidado para com o futuro", por exemplo) ao redor do conceito de "impactos apropriados". Até o momento, estes têm se centrado em grande medida ao redor de "pontos de referência" generalizáveis, como a Declaração Universal dos Direitos Humanos da ONU ou o Tratado sobre a União Europeia (von SCHOMBERG, 2013). Tais tentativas de universalização podem produzir, de forma inconveniente, parâmetros normativos "frágeis" e que podem mascarar, sob a guisa da universalidade, narrativas culturais específicas sobre o que o amplo espectro de atores chave, em contextos culturais diferentes, julgam ser os aspectos e resultados relevantes dos processos de inovação. A importância de tais considerações foi ressaltada, no decorrer do workshop, em discussões sobre o que é normalmente deixado de fora de processos quantitativos e abordagens contemporâneas de estimação de riscos. As reações emocionais que as pessoas têm às inovações (ou à ausência das mesmas), por exemplo, foram exemplificadas através da discussão de um dos participantes sobre como o Brasil foi historicamente alçado à categoria de "vilão do desmatamento". Dentro de abordagens e discursos ligados à IR existe a expectativa de que os agentes de inovação ajam de forma reflexiva e responsável, mas raramente é tomada em consideração a existência das capacidades e habilidades afetivas necessárias, ou de quais são os recursos culturais disponíveis para tal elaboração emocional. Fora do mundo ocidental, por exemplo, onde relações não instrumentais podem existir de forma preponderante, a IR pode ser expressa através de manifestações cotidianas de alegria ou tristeza, na forma de compaixão ou rebelião, plasmada em esperança ou desespero. Os contextos culturais e institucionais específicos nos quais os agentes de inovação atuam precisam ser conhecidos, de modo que se possa entender as formas através das quais tais maneiras de engajamento emocional podem se manifestar efetivamente ou serem aniquiladas.

Além disso, alguns participantes questionaram a abordagem na qual tecnologias e inovações são ou devem ser consideradas instrumentos para a materialização de metas e objetivos. As tecnologias são mediadoras, no decorrer do tempo, da forma como concebemos quais devem ser nossos objetivos, da mesma forma como são concebidas para terem impactos específicos na realidade (MOL, 2008). As tecnologias devem ser tratadas, ao invés disso, como elementos de práticas de cuidado que tanto servem a fins específicos quanto elementos que transformam nossa percepção destes mesmos fins. A adoção de uma abordagem como essa não apenas permite que se atue com sensibilidade no que diz respeito a diferenças culturais (e especialmente afetivas) nas quais a imaginação do futuro é constituída, mas também que o foco de atenção se estabeleça sobre aspectos mais relevantes do presente - particularmente sobre quais capacidades, disposições e virtudes são desejáveis e podem ser fomentadas por tecnologias específicas, ou como tais capacidades, disposições e virtudes irão transformar a forma como lidamos com um futuro intrinsecamente incerto.

\section{Conclusões}

A perspectiva aqui elaborada é oriunda das discussões ocorridas no âmbito do workshop, onde os participantes refletiram sobre a IR em um contexto de contrastes culturais, elaborados na forma de tensões, paradoxos e possibilidades. Gostaríamos de pontuar o que os pesquisadores britânicos de IR aprenderam no Brasil e levaram à Europa. Inicialmente, evidenciou-se a necessidade de reconhecimento do fato de que a IR se desenvolveu em um contexto sociopolítico especificamente europeu, com todas as implicações que advêm desse fato; segundo, reconheceu-se a necessidade de que a IR se engaje nos contextos, culturas e práticas locais (seja no Reino Unido, no Brasil ou em qualquer outro lugar), o que implica a consideração de formas locais de inovação social; terceiro, é necessário que a IR fomente um diálogo permanente e crítico com formas estabelecidas de inovação intensivas em capital, expondo irresponsabilidades sistêmicas e abrindo espaço para formas de inovação distintas, mais sustentáveis e justas; e quarto, o reconhecimento de que a IR não tem o objetivo de impor uma abordagem pré-formatada aos países do Sul mas, ao invés disso, utilizar seu poder de influência - na qualidade de abordagem com considerável peso político - para criar espaço para formas alternativas de desenvolvimento, complementando e estabelecendo diálogos com discursos locais estabelecidos.

Uma questão repetidamente levantada no workshop diz respeito ao tema da capacitação, com o debate sobre como construir e integrar a IR a práticas existente no mundo real. Há uma demanda crescente por ferramentas e orientação na construção de capacidades distribuídas em IR (por exemplo, a partir da Comissão Europeia, de cientistas que querem implementar a IR em suas solicitações de verbas e iniciativas de capacitação, etc.). A provisão de tal treinamento requer, em si mesma, os seguintes elementos: o desenvolvimento de novos currículos e sua difusão por contextos culturais e disciplinares diversos; o conhecimento dos papéis desempenhados por atores diferentes (e.g. engenheiros e cientistas naturais) nos processos de inovação; a criação de redes como modelos híbridos de gestão; e o desenvolvimento de indicadores, em setores e 
tecnologias distintos, de modo a exemplificar como a abordagem de IR pode ser utilizada em diferentes contextos nacionais e (inter)disciplinares.

Teorias e práticas de IR têm a desafortunada tendência de se separarem, ao passo que é evidente que ambas devem e podem informar umas às outras. Nos debates do workshop identificamos a necessidade real da manutenção do diálogo entre os avanços analíticos das pesquisas de IR e o desenvolvimento de ferramentas e práticas concretas na "execução" da IR. Igualmente, no que diz respeito à obtenção de resultados efetivos, reconheceu-se que teorias e dados empíricos, ainda que bem fundamentados, não são o suficiente. Tais ideias e as evidências a elas associadas precisam ser desenvolvidas, mas os efeitos só se farão sentir quando hábitos e rotinas institucionais, científicas e de gestão bem estabelecidas forem desarticuladas. Este é elemento central na manutenção e desenvolvimento de uma atitude crítica e reflexiva, tanto nos estudos de ciência e tecnologia de IR quanto em escalas mais amplas, a partir do momento em que a IR começa a expandir suas fronteiras.

Um dos objetivos formais do workshop era desenvolver ideias para colaborações futuras. Um grande número de projetos colaborativos encontra-se em desenvolvimento, em estágios diversos de execução, objetivando uma compreensão mais detalhada da eficácia da IR como discurso político emergente em arenas não-europeias. As ideias apresentadas neste artigo serão desenvolvidas mais adiante, na forma de uma edição especial do periódico Journal of Responsible Innovation.

Tradução: Marko Monteiro e Renzo Taddei.

Fonte de financiamento: O workshop teve o financiamento do British Council e da Fundação de Amparo à Pesquisa do Estado de São Paulo (FAPESP), apoio número 2013/50610-0 - FAPESP/British Council Researcher Links Workshop 2013 (BCRLW-13).

\section{Referências}

DA MATTA, R. Fé em Deus e pé na tábua - ou como e por que o trânsito enlouquece no Brasil. Rio de Janeiro: Rocco, 2010.

DAGNINO, R. Why science and technology capacity building for social development? Science \& Public Policy, v. 39, n. 5, p. 548-556, 2012.
FELT, U. et al. Taking European Knowledge Seriously. Brussels: European Commission, 2007. Report of the Expert Group on Science and Governance to the Science, Economy and Society Directorate, DirectorateGeneral for Research.

GOLDEMBERG, J́. Ethanol for a sustainable energy future. Science, v. 315, n. 5813, p. 808-810, 2007.

GUDYNAS, E. Buen Vivir: today's tomorrow. Development, v. 54, n. 4, p. 441-447, 2011.

GUPTA, A. Innovations for the Poor by the Poor. International Journal of Technological Learning, Innovation and Development, v. 5, n. 1/2, p. 28-39, 2012.

MICHENER, G. Brazil's growing car obsession. Observing Brazil, 2014. Disponível em: < http://observingbrazil. com/2012/03/04/brazils-growing-car-obsession>. Acesso em: 11 abr. 2014.

MOL, A. The logic of care: health and the problem of patient choice. New York: Routledge, 2008.

OWEN, R.; BESSANT, J.; HEINTZ, M. Responsible innovation: managing the responsible emergence of science and innovation in society. Chichester: Wiley, 2013.

OWEN, R.; MACNAGHTEN, P.; STILGOE, J. Responsible research and innovation: from science in society to science for society, with society. Science \& Public Policy, v. 39, n. 6, p. 751-760, 2012.

PRAHALAD, C. K. The fortune at the bottom of the pyramid. Upper Saddle River: Wharton School Publishing, 2006.

SORDA, G.; BANSE, M.; KEMFERT, C. An overview of biofuel policies across the world. Energy Policy, v. 38, n. 11, p. 6977-6988, 2010.

STILGOE, J.; OWEN, R.; MACNAGHTEN, P. Developing a framework of responsible innovation. Research Policy, v. 42, n. 9, p. 1568-1580, 2013.

VAN OUDHEUSDEN, M. Where are the politics in responsible innovation? European governance, technology assessments, and beyond. Journal of Responsible Innovation, v. 1, n. 1, p. 67-86, 2014.

VON SCHOMBERG, R. A Vision of responsible research and innovation. In: OWEN, R.; BESSANT, J.; HEINTZ, M. (Ed.). Responsible innovation: managing the responsible emergence of science and innovation in society. Chichester: Wiley, 2013. p. 51-74.

WALTER, A. et al. Sustainability assessment of bio-ethanol production in brazil considering land use change GHG emissions and socio-economic aspects. Energy Policy, v. 39, n. 10, p. 5703-5716, 2011. 